\title{
The Terminal Responses of the Two-Wire Line in Multiaperture Cavities Based on Electromagnetic Topology and Method of Moments
}

\author{
Ying Li, Jianshu Luo, and Guyan Ni \\ Department of Mathematics and System Science, College of Science, National University of \\ Defense Technology, Changsha, Hunan 410073, China \\ Correspondence should be addressed to Ying Li, liying801@163.com
}

Received 1 September 2010; Accepted 7 January 2011

Academic Editor: Alois Steindl

Copyright (C) 2011 Ying Li et al. This is an open access article distributed under the Creative Commons Attribution License, which permits unrestricted use, distribution, and reproduction in any medium, provided the original work is properly cited.

\begin{abstract}
A simulation technique based on electromagnetic topology (EMT) theory is proposed for analyzing electromagnetic interference (EMI) coupling through apertures onto the two-transmission line enclosed within metallic structures. The electromagnetic interactions between apertures and the external-internal interactions were treated through the topological decomposition and the multistep iterative method. Then, the load responses of the two-wire transmission line are resolved by the the Baum-Liu-Tesche (BLT) equation. The simulation results both without and with the electromagnetic interaction are presented for the frequency range from $100 \mathrm{MHz}$ to $3 \mathrm{GHz}$. These numerical results obtained by two methods imply that the electromagnetic interaction cannot be simply ignored, especially for the frequency range up to $1 \mathrm{GHz}$.
\end{abstract}

\section{Introduction}

While considering analysis and design of EMC, it is important to protect electronic circuits/components from electromagnetic effects due to external illumination. For this purpose, electronic circuits/components are often shielded inside metallic cavities. However, these metallic surfaces are not perfect. On a metallic cavity, there may be apertures that become sources of the EMI problem. In most cases the cavity and apertures are usually rectangular, which has led to a number of attempts to solve the coupling problem of the rectangular cavity with a series of rectangular apertures when illuminated by a plane wave (see [1-3]). So there would be interference between the fields entering through the apertures and the circuitry inside the cavities. A natural problem is a comprehensive analysis of the coupling mechanism for such structures under external EMI illumination. In this paper, electromagnetic interaction problems can be simulated through codes based on electromagnetic topology. An important element of topological analysis is the determination 


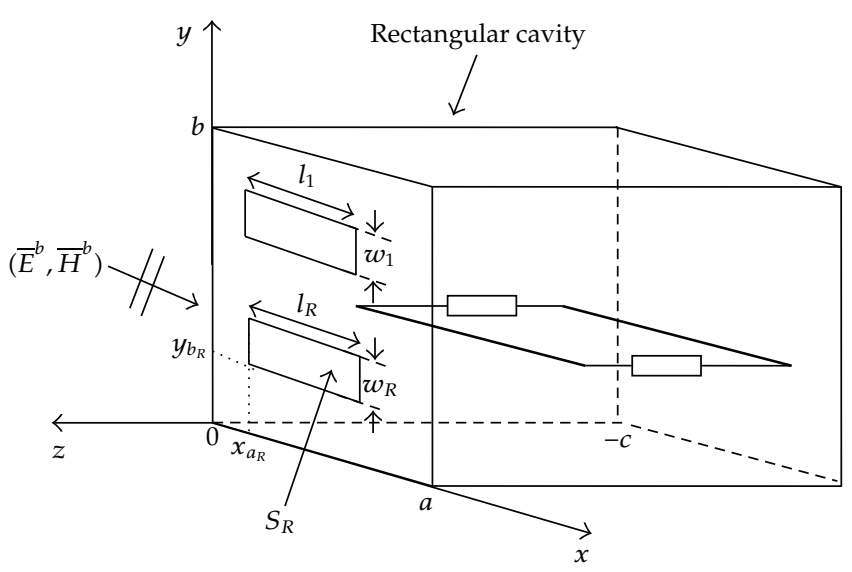

Figure 1: The rectangular cavity with $R$ rectangular apertures.

of a mechanism which represents the external-internal coupling through a small aperture and the subsequent propagation process. By combining methodologies suggested earlier (see [4]), an equivalent source can be created to relate the electromagnetic coupling at the exterior surface and the transfer function through the free space by assuming an imaginary transmission line as a source of the aperture radiation (see $[5,6]$ ), but this method only adapts to the case where the dimension of the aperture is electrically small when compared to the wavelength. Moreover most papers in the literature neglect the electromagnetic interaction among apertures (see $[3,7]$ ), which is not reasonable.

This paper provides a novel numerical technique for the electromagnetic coupling. More precisely, we first use the EMT theory and the multistep iteration to deal with problems of coupling between apertures and the external-internal interaction. Then, we employ dyadic Green's functions and the method of moments to determine the electromagnetic coupling fields inside the cavity. Finally, we apply the BLT equation to resolve the load response of the two-wire transmission line. The simulation result shows the effect of the external coupling fields on the two-wire line current depends on both the cavity with multiaperture and the electromagnetic interaction.

\section{The Topological Decomposition of Complicated Electrical Systems}

Electromagnetic interaction problems on very large and complex system, such as an aircraft, can be simulated through codes based on the EMT. The most important aspect of the EMT is the assumption that volumes can be decomposed into subvolumes that can be interacted with each other through apertures (see [8]). To analyze the interaction processes for the electromagnetic coupling by the EMT, we need to establish the topological structure model and the topological diagram for the system configuration. First, we consider a rectangular cavity with $R$ energy penetration paths (multiaperture) illuminated by a harmonic plane wave, see Figure 1. Figure 2 shows the topological structure model associated to Figure 1. $V_{i}$ denote these subvolumes, where " $i$ " indicates the hierarchical order of the volume. $S_{i, j}$ is a surface separating volumes $V_{i}$ from $V_{j}$. Figure 3 shows the topological diagram. To analyze the field coupling phenomenon in the cavity, we introduce a "field coupling junction" $J_{3}$, which takes into account the internal EM field coupling to the two-wire line. Moreover, by 


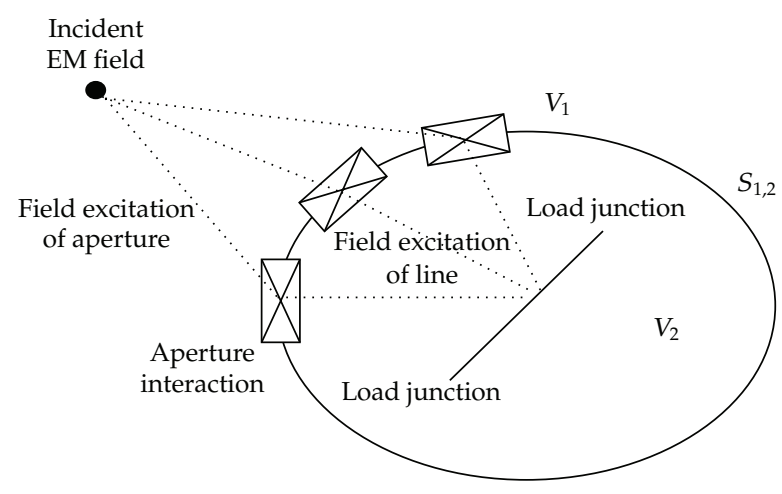

Figure 2: The system topological structure model.

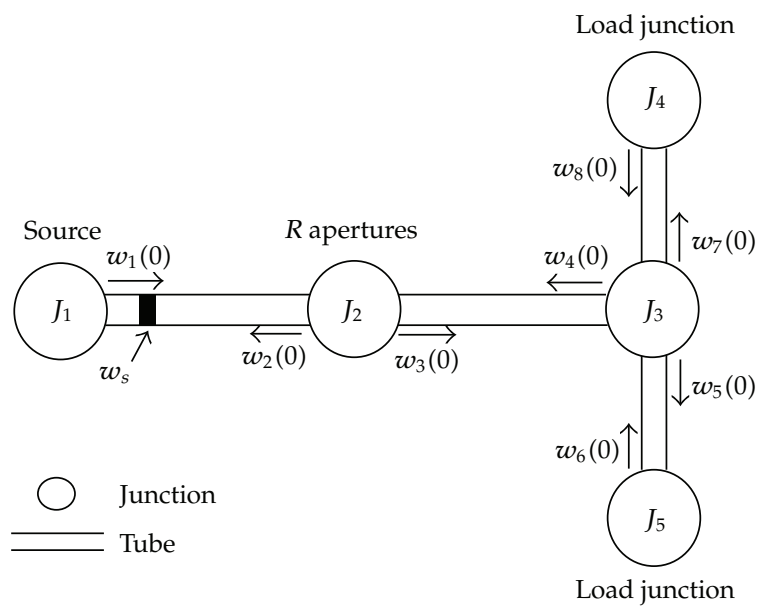

Figure 3: The topology diagram of Figure 2.

reciprocity, this junction also provides effects of the EM field radiation by the two-wire line (see [9]). $W_{i}(0)$ is the outgoing wave and $W_{s}$ is the network cable coupling source. Signals on an entire transmission line network is expressed through the BLT (Baum-Liu-Tesche) equation which is the multiconductor transmission line (MTL) network composed of the outgoing wave supervector $[W(0)]$ and the source wave supervector $\left[W_{s}\right]$ (see [10]).

\section{The Multistep Iterative Method}

Based on the topology decomposition, we deal with problems of the electromagnetic interaction among apertures and the external-internal interaction using the multistep iterative method (see [11]). We assume $R$ apertures on the same wall located on the plane where $z=0 . R$ is the number of apertures.

Step 1 . Find the zeroth-order approximation for $R$-aperture magnetic currents $\bar{M}_{0}$ and the corresponding coupling electromagnetic fields $\bar{E}_{0}, \bar{H}_{0}$ in a cavity due to $\bar{M}_{0}$. 
Theoretically, a plane wave excitation represents the simplest electromagnetic source and therefore is particularly suitable to test numerical techniques. In this paper, without lose of practical applications, we will use a harmonic plane wave with a wide frequency spectrum to simplify the problem under consideration. Consider a rectangular cavity with $R$ rectangular apertures illuminated by a harmonic plane wave shown in Figure 1. This field is described by angles of incidence $\psi$ and $\phi$, as well as a polarization angles $\alpha$, which defines the E-field vector direction with respect to the vertical plane of the incidence. It is given as

$$
\begin{gathered}
\bar{H}^{i}=\left(H_{0 x} \widehat{x}+H_{0 y} \widehat{y}+H_{0 z} \widehat{z}\right) e^{-j k_{0} \hat{k}^{i} \cdot \bar{r}}, \\
\bar{E}^{i}=Z_{0} \bar{H}^{i} \times \widehat{k}^{i},
\end{gathered}
$$

where $\bar{r}=x \hat{x}+y \widehat{y}+z \widehat{z}$ refers to the location of the field in the free space,

$$
\widehat{k}^{i}=-(\sin \theta \cos \phi \widehat{x}+\sin \theta \sin \phi \widehat{y}+\cos \theta \widehat{z})
$$

$k_{0}=\omega \sqrt{\mu_{0} \varepsilon_{0}}$ is the free space wave number, and $Z_{0}$ is the free space intrinsic impedance. For a unity amplitude electric field, the coefficients $H_{0 x}, H_{0 y}, H_{0 z}$ are given by

$$
\begin{gathered}
H_{0 x}=\frac{1}{Z_{0}}(\sin \alpha \cos \theta \cos \phi+\cos \alpha \sin \phi), \\
H_{0 y}=\frac{1}{Z_{0}}(\sin \alpha \cos \theta \sin \phi-\cos \alpha \cos \phi), \\
H_{0 z}=-\frac{1}{Z_{0}} \sin \alpha \sin \theta .
\end{gathered}
$$

We introduce equivalent magnetic currents $\bar{M}_{0}$ on $R$ apertures as

$$
\bar{M}_{0}=\sum_{r=1}^{R} \bar{M}_{0 r}=\sum_{r=1}^{R}\left[M_{0 r x}(x, y) \widehat{x}+M_{0 r y}(x, y) \widehat{y}\right]
$$

where $\bar{M}_{0 r}$ is an equivalent magnetic current on the $r$ th aperture.

The equivalent magnetic current components now are expanded as

$$
\begin{aligned}
& M_{0 r x}(x, y)=\sum_{p=1}^{P_{r}-1} \sum_{q=1}^{Q_{r}} M_{0 r x p q} \Psi_{r p q}(x, y), \\
& M_{0 r y}(x, y)=\sum_{p=1}^{P_{r}} \sum_{q=1}^{Q_{r}-1} M_{0 r y p q} \Phi_{r p q}(x, y),
\end{aligned}
$$


where

$$
\begin{aligned}
& \Psi_{r p q}(x, y)=T_{p}\left(x-x_{a_{r}}\right) P_{q}\left(y-y_{b_{r}}\right), \\
& \Phi_{r p q}(x, y)=P_{p}\left(x-x_{a_{r}}\right) T_{q}\left(y-y_{b_{r}}\right),
\end{aligned}
$$

in which $\left(x_{a_{r}}, y_{b_{r}}\right)$ is the coordinates of the lower left-hand corner of the $r$ th aperture, $(p, q)$ are integers used for multiaperture modes, $T_{p}(t)$ and $P_{q}(t)$ are triangular and pulse functions defined by

$$
T_{p}(t)= \begin{cases}\frac{t-(p-1) \Delta t}{\Delta t}, & (p-1) \Delta t \leq t \leq p \Delta t, \\ \frac{(p+1) \Delta t-t}{\Delta t}, & p \Delta t \leq t \leq(p+1) \Delta t \\ 0, & |t-p \Delta t| \geq \Delta t\end{cases}
$$

for $p=1,2, \ldots, P_{r}-1$, and

$$
P_{q}(t)= \begin{cases}1, & (q-1) \Delta t \leq t \leq q \Delta t \\ 0, & \text { otherwise, }\end{cases}
$$

for $q=1,2, \ldots, Q_{r}$.

Substituting (3.7) into expression (3.6), we derive equivalent magnetic currents as

$$
\bar{M}_{0}=\sum_{r=1}^{R}\left[\sum_{p=1}^{P_{r}-1} \sum_{q=1}^{Q_{r}} M_{0 r x p q} \Psi_{r p q}(x, y) \widehat{x}+\sum_{p=1}^{P_{r}} \sum_{q=1}^{Q_{r}-1} M_{0 r y p q} \Phi_{r p q}(x, y) \widehat{y}\right] .
$$

To solve the unknown currents $\bar{M}_{0}$, we use the continuity of tangential magnetic fields across $R$ apertures. Then, we have

$$
\widehat{z} \times\left[\bar{H}^{a}\left(\bar{M}_{0}\right)+\bar{H}^{i}\right]=-\widehat{z} \times \bar{H}^{b}\left(\bar{M}_{0}\right), \quad z=0,
$$

where $\bar{H}^{a}$ refers to the corresponding exterior scattered magnetic field due to $\bar{M}_{0}$, and $\bar{H}^{b}$ refers to the corresponding interior coupling magnetic field due to $\bar{M}_{0}$.

The exterior scattered field can be expressed as the radiation caused by the equivalent magnetic current $\bar{M}_{0}$, say,

$$
\bar{H}^{a}\left(\bar{M}_{0}\right)=-j k_{0} Y_{0} \int_{\sum_{r=1}^{R} S_{r}} 2 \bar{M}_{0}\left(\bar{r}^{\prime}\right) \cdot \Gamma_{0}\left(\bar{r} ; \bar{r}^{\prime}\right) d s^{\prime}
$$


where $S_{r}$ denotes the surface of the $r$ th aperture and $\Gamma_{0}\left(\bar{r} ; \bar{r}^{\prime}\right)$ is the dyadic Green's function in the free space, which reads

$$
\begin{gathered}
\Gamma_{0}\left(\bar{r} ; \bar{r}^{\prime}\right)=\left(I+\left(\frac{1}{k_{0}^{2}}\right) \nabla \nabla\right) \frac{e^{-j k_{0}\left|\bar{r}-\bar{r}^{\prime}\right|}}{4 \pi\left|\bar{r}-\bar{r}^{\prime}\right|} \\
\left|\bar{r}-\bar{r}^{\prime}\right|=\sqrt{\left(x-x^{\prime}\right)^{2}+\left(y-y^{\prime}\right)^{2}}
\end{gathered}
$$

in which $\bar{r}$ and $\bar{r}^{\prime}$ represent the locations of both the field and source points on the aperture, respectively.

The interior field can be formulated as the radiation due to $\bar{M}_{0}$ on $R$ apertures. Using the available dyadic Green's functions for the cavity, we can express the interior field as

$$
\begin{aligned}
& \bar{E}^{b}\left(\bar{M}_{0}\right)=\int_{\sum_{r=1}^{R} S_{r}} \nabla \times G_{H M} \cdot \bar{M}_{0} d s^{\prime}, \\
& \bar{H}^{b}\left(\bar{M}_{0}\right)=j \omega \varepsilon \int_{\sum_{r=1}^{R} S_{r}} G_{H M} \cdot \bar{M}_{0} d s^{\prime},
\end{aligned}
$$

where the dyadic Green's function is defined as

$$
\begin{aligned}
G_{H M}= & -\frac{1}{k_{0}^{2}} \widehat{z} \widehat{z} \delta\left(\bar{R}-\bar{R}^{\prime}\right)-\sum_{m, n} \frac{2\left(2-\delta_{m n}\right)}{a b k_{c}^{2} k_{m n} \sin \left(k_{m n} c\right)} \\
& \cdot\left[\bar{M}_{o e}(z+c) \bar{M}_{o e}^{\prime}(0)+\bar{N}_{e o}(z+c) \bar{N}_{e o}^{\prime}(0)\right]
\end{aligned}
$$

where $\delta_{m n}$ denotes the Kronecker delta, say, $\delta_{m n}=1$ for $m$ or $n=0$, and zero otherwise. Also, the wave functions within representation (3.18) are given by

$$
\begin{aligned}
\bar{M}_{o e}(z)= & k_{y n} \sin \left(k_{x m} x\right) \cos \left(k_{y n} y\right) \cos \left(k_{m n} z\right) \hat{x} \\
& -k_{x m} \cos \left(k_{x m} x\right) \sin \left(k_{y n} y\right) \cos \left(k_{m n} z\right) \hat{y}, \\
\bar{N}_{e o}(z)=\frac{1}{k_{b}}[ & -k_{m n} k_{x m} \sin \left(k_{x m} x\right) \cos \left(k_{y n} y\right) \cos \left(k_{m n} z\right) \hat{x} \\
& -k_{m n} k_{y n} \cos \left(k_{x m} x\right) \sin \left(k_{y n} y\right) \cos \left(k_{m n} z\right) \hat{y} \\
& \left.+k_{c}^{2} \cos \left(k_{x m} x\right) \cos \left(k_{y n} y\right) \sin \left(k_{m n} z\right) \hat{z}\right] .
\end{aligned}
$$

As usual, $k_{b}=k_{0} \sqrt{\mu_{b} \varepsilon_{b}}=\omega \sqrt{\mu \varepsilon} ; k_{x m}=m \pi / a, k_{y n}=n \pi / b, k_{c}^{2}=k_{x m}^{2}+k_{y n}^{2}$, and

$$
k_{m n}= \begin{cases}-j \sqrt{k_{c}^{2}-k_{0}^{2}}, & k_{0}^{2}<k_{c}^{2} \\ \sqrt{k_{0}^{2}-k_{c}^{2}}, & k_{0}^{2}>k_{c}^{2}\end{cases}
$$

with $m, n$ being nonnegative integers excluding $m=n=0$. 
Substituting (3.18) into expressions (3.16) and (3.17) yields all components of the electromagnetic field inside the cavity as

$$
\begin{aligned}
& E_{0 x}^{b}(x, y, z)=-\sum_{r=1}^{R} \sum_{m, n} \frac{2\left(2-\delta_{m n}\right)}{a b \sin \left(k_{m n} c\right)} \sum_{p=1}^{P_{r}} \sum_{q=1}^{Q_{r}-1} M_{0 r y p q} I_{r y p q m n} \\
& \cdot \cos \left(k_{x m} x\right) \sin \left(k_{y n} y\right) \sin \left(k_{m n}(z+c)\right) \text {, } \\
& E_{0 y}^{b}(x, y, z)=\sum_{r=1}^{R} \sum_{m, n} \frac{2\left(2-\delta_{m n}\right)}{a b \sin \left(k_{m n} c\right)} \sum_{p=1}^{P_{r}-1} \sum_{q=1}^{Q_{r}} M_{0 r x p q} I_{r x p q m n} \\
& \cdot \sin \left(k_{x m} x\right) \cos \left(k_{y n} y\right) \sin \left(k_{m n}(z+c)\right) \text {, } \\
& E_{0 z}^{b}(x, y, z)=-\sum_{r=1}^{R} \sum_{m, n} \frac{2\left(2-\delta_{m n}\right)}{a b k_{m n} \sin \left(k_{m n} c\right)} \\
& \cdot\left[k_{y n} \sum_{p=1}^{P_{r}-1} \sum_{q=1}^{Q_{r}} M_{0 r x p q} I_{r x p q m n}-k_{x m} \sum_{p=1}^{P_{r}} \sum_{q=1}^{Q_{r}-1} M_{0 r y p q} I_{r y p q m n}\right] \\
& \cdot \sin \left(k_{x m} x\right) \sin \left(k_{y n} y\right) \cos \left(k_{m n}(z+c)\right) \text {, } \\
& H_{0 x}^{b}(x, y, z)=-j Y_{b} \sum_{r=1}^{R} \sum_{m, n} \frac{2\left(2-\delta_{m n}\right)}{a b k_{m n} k_{b} \sin \left(k_{m n} c\right)} \\
& \cdot\left[\left(k_{b}^{2}-k_{x m}^{2}\right) \sum_{p=1}^{P_{r}-1} \sum_{q=1}^{Q_{r}} M_{0 r x p q} I_{r x p q m n}+\left(-k_{x m} k_{y n}\right) \sum_{p=1}^{P_{r}} \sum_{q=1}^{Q_{r}-1} M_{0 r y p q} I_{r y p q m n}\right] \\
& \cdot \sin \left(k_{x m} x\right) \cos \left(k_{y n} y\right) \cos \left(k_{m n}(z+c)\right), \\
& H_{0 y}^{b}(x, y, z)=-j Y_{b} \sum_{r=1}^{R} \sum_{m, n} \frac{2\left(2-\delta_{m n}\right)}{a b k_{m n} k_{b} \sin \left(k_{m n} c\right)} \\
& \cdot\left[\left(-k_{x m} k_{y n}\right) \sum_{p=1}^{P_{r}-1} \sum_{q=1}^{Q_{r}} M_{0 r x p q} I_{r x p q m n}+\left(k_{b}^{2}-k_{y n}^{2}\right) \sum_{p=1}^{P_{r}} \sum_{q=1}^{Q_{r}-1} M_{0 r y p q} I_{r y p q m n}\right] \\
& \cdot \cos \left(k_{x m} x\right) \sin \left(k_{y n} y\right) \cos \left(k_{m n}(z+c)\right), \\
& H_{0 z}^{b}(x, y, z)=j Y_{b} \sum_{r=1}^{R} \sum_{m, n} \frac{2\left(2-\delta_{m n}\right)}{a b k_{b} \sin \left(k_{m n} c\right)} \\
& \cdot\left[k_{x m} \sum_{p=1}^{P_{r}-1} \sum_{q=1}^{Q_{r}} M_{0 r x p q} I_{r x p q m n}+k_{y n} \sum_{p=1}^{P_{r}} \sum_{q=1}^{Q_{r}-1} M_{0 r y p q} I_{r y p q m n}\right] \\
& \cdot \cos \left(k_{x m} x\right) \cos \left(k_{y n} y\right) \sin \left(k_{m n}(z+c)\right) \text {, }
\end{aligned}
$$


where $Y_{b}=1 / Z_{b}=\left(1 / Z_{0}\right) \sqrt{\varepsilon_{b} / \mu_{b}}=\sqrt{\varepsilon / \mu}, k_{b}=k_{0} \sqrt{\mu_{b} \varepsilon_{b}}=\omega \sqrt{\mu \varepsilon}$, and

$$
\begin{aligned}
I_{r x p q m n}= & \frac{8 \sin ^{2}\left(k_{x m}\left(\Delta x_{r} / 2\right)\right) \sin \left(k_{y n}\left(\Delta y_{r} / 2\right)\right)}{k_{x m}^{2} k_{y n} \Delta x_{r}} \\
& \cdot \sin \left[k_{x m}\left(x_{a_{r}}+p \Delta x_{r}\right)\right] \cos \left[k_{y n}\left(y_{b_{r}}+\left(q-\frac{1}{2}\right) \Delta y_{r}\right)\right], \\
I_{r y p q m n}= & \frac{8 \sin ^{2}\left(k_{y n}\left(\Delta y_{r} / 2\right)\right) \sin \left(k_{x m}\left(\Delta x_{r} / 2\right)\right)}{k_{y n}^{2} k_{x m} \Delta y_{r}} \\
& \cdot \sin \left[k_{y n}\left(y_{b_{r}}+q \Delta y_{r}\right)\right] \cos \left[k_{x m}\left(x_{a_{r}}+\left(p-\frac{1}{2}\right) \Delta x_{r}\right)\right] .
\end{aligned}
$$

$M_{0 r x p q}$ and $M_{0 r y p q}$ are the unknown constants of the $p q$ mode on the $r$ th aperture, $w_{r}, l_{r}$ are the width and length of the $r$ th aperture, respectively. $\Delta x_{r}, \Delta y_{r}$ refer to the width and length of the surface element used to discretize the $r$ th aperture.

$M_{0 \text { rypq }}$ is

Using Galerkin's method (see [12]), the integral equation to be solved for $M_{0 r x p q}$,

$$
\int_{\sum_{r=1}^{R} S_{r}} \hat{z} \times\left[\bar{H}^{a}\left(\bar{M}_{0}\right)+\bar{H}^{b}\left(\bar{M}_{0}\right)\right] \cdot \bar{W} d s^{\prime}=-\int_{\sum_{r=1}^{R} S_{r}} \hat{z} \times \bar{H}^{i} \cdot \bar{W} d s^{\prime}, \quad z=0,
$$

where $\bar{W}$ is a weighting function, $\bar{H}^{a}\left(\bar{M}_{0}\right)$ and $\bar{H}^{b}\left(\bar{M}_{0}\right)$ are given by (3.14) and (3.17), respectively. To discretize (3.23), the corresponding weighing functions are given by

$$
\bar{W}_{r^{\prime} p^{\prime} q^{\prime}}=\Phi_{r^{\prime} p^{\prime} q^{\prime}}(x, y) \hat{x}+\Psi_{r^{\prime} p^{\prime} q^{\prime}}(x, y) \hat{y} \text {. }
$$

where $\Phi_{r^{\prime} p^{\prime} q^{\prime}}(x, y)$ and $\Psi_{r^{\prime} p^{\prime} q^{\prime}}(x, y)$ are given by (3.8) and (3.9), respectively. Substitution of (3.14) and (3.17) into (3.23) yields a matrix equation:

$$
\left[Y^{a+b}\right]\left[M_{0}\right]=\left[C^{\mathrm{inc}}\right] \text {. }
$$

The expressions of all matrix entries in (3.25) are as follows.

Self-admittance matrix $\left[Y^{b}\right]$ is represented by

$$
\begin{aligned}
& \left(Y^{b_{11}}\right)_{r p q r^{\prime} p^{\prime} q^{\prime}}=\frac{j \omega \varepsilon_{0}}{k_{b}^{2}} \sum_{m, n} \frac{\varepsilon_{m} \varepsilon_{n}\left(k_{b}^{2}-k_{x m}^{2}\right)}{a b k_{m n} \tan \left(k_{m n} c\right)} I_{r x p q m n} I_{r^{\prime} x p^{\prime} q^{\prime} m n} \\
& \left(Y^{b_{12}}\right)_{r p q r^{\prime} p^{\prime} q^{\prime}}=\frac{j \omega \varepsilon_{0}}{k_{b}^{2}} \sum_{m, n} \frac{\varepsilon_{m} \varepsilon_{n} k_{x m} k_{y n}}{a b k_{m n} \tan \left(k_{m n} c\right)} I_{r y p q m n} I_{r^{\prime} x p^{\prime} q^{\prime} m n} \\
& \left(Y^{b_{21}}\right)_{r p q r^{\prime} p^{\prime} q^{\prime}}=\frac{j \omega \varepsilon_{0}}{k_{b}^{2}} \sum_{m, n} \frac{\varepsilon_{m} \varepsilon_{n} k_{x m} k_{y n}}{a b k_{m n} \tan \left(k_{m n} c\right)} I_{r x p q m n} I_{r^{\prime} y p^{\prime} q^{\prime} m n} \\
& \left(Y^{b_{22}}\right)_{r p q r^{\prime} p^{\prime} q^{\prime}}=\frac{j \omega \varepsilon_{0}}{k_{b}^{2}} \sum_{m, n} \frac{\varepsilon_{m} \varepsilon_{n}\left(k_{b}^{2}-k_{y n}^{2}\right)}{a b k_{m n} \tan \left(k_{m n} c\right)} I_{r y p q m n} I_{r^{\prime} y p^{\prime} q^{\prime} m n}
\end{aligned}
$$

where $\varepsilon_{m}$ is Neumann's numbers, and $I_{\text {rxpqmn }}, I_{\text {rypqmn }}$ are given by (3.22). 
External admittance matrix $\left[Y^{a}\right]$ is represented by

$$
\begin{aligned}
& \left(Y^{a_{11}}\right)_{r p q r^{\prime} p^{\prime} q^{\prime}}=-\frac{\omega \varepsilon_{0}}{4\left(\pi k_{0}\right)^{2}} \iint_{-\infty}^{+\infty} \frac{\left(k_{0}^{2}-k_{x m}^{2}\right)}{k_{m n}} F_{r p q} F_{r p q}^{*} d k_{x m} d k_{y n} \\
& \left(Y^{a_{12}}\right)_{r p q r^{\prime} p^{\prime} q^{\prime}}=-\frac{\omega \varepsilon_{0}}{4\left(\pi k_{0}\right)^{2}} \iint_{-\infty}^{+\infty} \frac{k_{x m} k_{y n}}{k_{m n}} G_{r p q} F_{r^{\prime} p^{\prime} q^{\prime}}^{*} d k_{x m} d k_{y n} \\
& \left(Y^{a_{21}}\right)_{r p q r^{\prime} p^{\prime} q^{\prime}}=-\frac{\omega \varepsilon_{0}}{4\left(\pi k_{0}\right)^{2}} \iint_{-\infty}^{+\infty} \frac{k_{x m} k_{y n}}{k_{m n}} F_{r p q} G_{r^{\prime} p^{\prime} q^{\prime}}^{*} d k_{x m} d k_{y n} \\
& \left(Y^{a_{22}}\right)_{r p q r^{\prime} p^{\prime} q^{\prime}}=-\frac{\omega \varepsilon_{0}}{4\left(\pi k_{0}\right)^{2}} \iint_{-\infty}^{+\infty} \frac{\left(k_{0}^{2}-k_{x m}^{2}\right)}{k_{m n}} G_{r p q} G_{r p q}^{*} d k_{x m} d k_{y n},
\end{aligned}
$$

where $F_{r p q}\left(k_{x m}, k_{y n}\right)$ and $G_{r p q}\left(k_{x m}, k_{y n}\right)$ are the Fourier transforms of $\Psi_{r p q}(x, y)$ and $\Phi_{r p q}(x, y)$, respectively. By replacing $k_{x m}, k_{y n}$ by $-k_{x m},-k_{y n}$, the expressions $F_{r p q}^{*}$ and $G_{r p q}^{*}$ can be obtained from expressions of $F_{r p q}$ and $G_{r p q}$, respectively.

Admittance matrix $\left[Y^{a+b}\right]$ is represented by

$$
\left[Y^{a+b}\right]=\left[Y^{a}\right]+\left[Y^{b}\right]
$$

Excitation vector $\left[C^{\text {inc }}\right]$ is represented by

$$
\begin{aligned}
\left(C_{x}^{\text {inc }}\right)_{r p q}= & -2 H_{0 x} \Delta x_{r} \Delta y_{r} e^{j k_{0} \sin \theta\left[p \Delta x_{r}+x_{a_{r}}\right] \cos \phi+\left[(q-1 / 2) \Delta y_{r}+y_{b_{r}}\right] \sin \phi} \\
& \cdot \operatorname{sinc}^{2}\left(\frac{k_{0} \Delta x_{r} \sin \theta \cos \phi}{2}\right) \operatorname{sinc}\left(\frac{k_{0} \Delta y_{r} \sin \theta \sin \phi}{2}\right), \\
\left(C_{y}^{\text {inc }}\right)_{r p q}= & -2 H_{0 y} \Delta x_{r} \Delta y_{r} e^{j k_{0} \sin \theta\left[(p-1 / 2) \Delta x_{r}+x_{a_{r}}\right] \cos \phi+\left[q \Delta y_{r}+y_{b_{r}}\right] \sin \phi} \\
& \cdot \operatorname{sinc}\left(\frac{k_{0} \Delta x_{r} \sin \theta \cos \phi}{2}\right) \operatorname{sinc}^{2}\left(\frac{k_{0} \Delta y_{r} \sin \theta \sin \phi}{2}\right),
\end{aligned}
$$

where $\operatorname{sinc}(x)=\sin (x) / x, H_{0 x}$ and $H_{0 y}$ are given by (3.3) and (3.4), respectively.

The solution of this matrix equation (3.25) yields the coefficients $M_{0 r x p q}, M_{0 r y p q}$ of $R$-aperture magnetic currents $\bar{M}_{0}$. A similar result can be found in $[3,11]$.

Step 2. Find $R$-aperture magnetic current increment $\Delta \bar{M}_{1}$ due to $\bar{E}_{0}^{b}, \bar{H}_{0}^{b}$ and find the corresponding exterior scatter fields $\Delta \bar{E}_{1}^{a}, \Delta \bar{H}_{1}^{a}$ and interior coupling fields $\Delta \bar{E}_{1}^{b}, \Delta \bar{H}_{1}^{b}$ due to $\Delta \bar{M}_{1}$.

To solve the unknown magnetic current increment $\Delta \bar{M}_{1}$, we require the tangential magnetic field be continuous in the following sense:

$$
\int_{\sum_{r=1}^{R} S_{r}} \hat{z} \times\left[\bar{H}^{b}\left(\bar{M}_{0}\right)+\bar{H}^{b}\left(\Delta \bar{M}_{1}\right)\right] \cdot \bar{W} d s=-\int_{\sum_{r=1}^{R} s_{r}} \hat{z} \times \bar{H}^{a}\left(\Delta \bar{M}_{1}\right) \cdot \bar{W} d s,
$$


where

$$
\begin{gathered}
\bar{H}^{b}\left(\Delta \bar{M}_{1}\right)=j \omega \varepsilon \int_{\sum_{r=1}^{R} S_{r}} G_{H M} \cdot \Delta \bar{M}_{1} d s^{\prime}, \\
\bar{H}^{a}\left(\Delta \bar{M}_{1}\right)=-j k_{0} Y_{0} \int_{\sum_{r=1}^{R} S_{r}} 2 \Delta \bar{M}_{1}\left(\bar{r}^{\prime}\right) \cdot \Gamma_{0}\left(\bar{r} ; \bar{r}^{\prime}\right) d s^{\prime},
\end{gathered}
$$

$\bar{H}^{b}\left(\bar{M}_{0}\right)$ and $\bar{W}$ are given by (3.17) and (3.24), respectively. Substitution of (3.17), (3.24), and (3.31) into (3.30) yields a matrix equation:

$$
\left[Y^{a+b}\right]\left[\Delta M_{1}\right]=-\left[Y^{b}\right]\left[M_{0}\right]
$$

The solution of this matrix equation (3.32) will yield the coefficients $\Delta M_{1 r x p q}, \Delta M_{1 r y p q}$ of the magnetic current increment $\Delta \bar{M}_{1}$.

Step 3. Find $R$-aperture magnetic current increment $\Delta \bar{M}_{2}$ due to $\Delta \bar{E}_{1}^{a}, \Delta \bar{H}_{1}^{a}$ and find the corresponding exterior scatter fields $\Delta \bar{E}_{2}^{a}, \Delta \bar{H}_{2}^{a}$ and internal coupling fields $\Delta \bar{E}_{2}^{b}, \Delta \bar{H}_{2}^{b}$ due to $\Delta \bar{M}_{2}$.

Similarly,

$$
\int_{\sum_{r=1}^{R} S_{r}} \widehat{z} \times \bar{H}^{b}\left(\Delta \bar{M}_{2}\right) \cdot \bar{W} d s=-\int_{\sum_{r=1}^{R} S_{r}} \widehat{z} \times\left[\bar{H}^{a}\left(\Delta \bar{M}_{1}\right)+\bar{H}^{a}\left(\Delta \bar{M}_{2}\right)\right] \cdot \bar{W} d s,
$$

and a matrix equation is obtained as

$$
\left[Y^{a+b}\right]\left[\Delta M_{2}\right]=-\left[Y^{a}\right]\left[\Delta M_{1}\right]
$$

So the solution of this matrix equation (3.34) will yield the coefficients $\Delta M_{2 \text { rxpq }}, \Delta M_{2 \text { rypq }}$ of the magnetic current increment $\Delta \bar{M}_{2}$.

Step 4. Find $R$-aperture magnetic current increment $\Delta \bar{M}_{3}$ due to $\Delta \bar{E}_{2}^{b}, \Delta \bar{H}_{2}^{b}$ and find the corresponding exterior scatter fields $\Delta \bar{E}_{3}^{a}, \Delta \bar{H}_{3}^{a}$ and internal coupling fields $\Delta \bar{E}_{3}^{b}, \Delta \bar{H}_{3}^{b}$ due to $\Delta \bar{M}_{3}$.

Similarly,

$$
\int_{\sum_{r=1}^{R} S_{r}} \widehat{z} \times\left[\bar{H}^{b}\left(\Delta \bar{M}_{2}\right)+\bar{H}^{b}\left(\Delta \bar{M}_{3}\right)\right] \cdot \bar{W} d s=-\int_{\sum_{r=1}^{R} S_{r}} \widehat{z} \times \bar{H}^{a}\left(\Delta \bar{M}_{3}\right) \cdot \bar{W} d s,
$$

and a matrix equation is obtained as:

$$
\left[Y^{a+b}\right]\left[\Delta M_{3}\right]=-\left[Y^{b}\right]\left[\Delta M_{2}\right]
$$




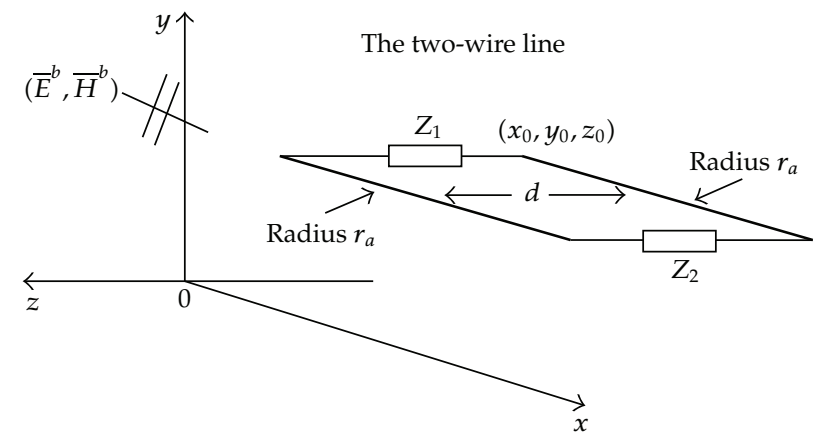

Figure 4: The isolated two-wire line excited by the interior coupling field.

So the solution of this matrix equation (3.36) will yield the coefficients $\Delta M_{3 r x p q}, \Delta M_{3 r y p q}$ of the magnetic current increment $\Delta \bar{M}_{3}$, and so on.

Finally, this iterative process approximates the magnetic currents on $R$ apertures and the total coupling electromagnetic field inside the cavity as

$$
\begin{aligned}
& \bar{M}=\bar{M}_{0}+\sum_{i=1}^{N_{\text {iter }}} \Delta \bar{M}_{i,} \\
& \bar{E}^{b}=\bar{E}_{0}^{b}+\sum_{i=1}^{N_{\text {iter }}} \Delta \bar{E}_{i}^{b} \\
& \bar{H}^{b}=\bar{H}_{0}^{b}+\sum_{i=1}^{N_{\text {iter }}} \Delta \bar{H}_{i}^{b} .
\end{aligned}
$$

We must point out that the four-step algorithm admits high accuracy for the approximation to the interaction among apertures and the external-internal interaction.

\section{The Application of the BLT Equation}

The EMT can simulate the response of inner circuits of the electrically large complex system. The BLT equation is the key equation of the EMT to express the signals on the entire transmission line network [13]. Hence we use the BLT equation to solve the terminal responses of the two-wire line in a multiaperture cavity illuminated by a plane wave. We consider the case where a lossless two-wire line is illuminated by a coupling EM field. In order to simplify the notation, let us place the two-wire transmission line in the plane where $y=y_{0}$, which parallels to the $x$-axis as shown in Figure 4 . We put the reference wire at the plane where $z=z_{0}$, and the other wire at $z=z_{0}+d$ so that there is distance $d$ between two wires. We assume that $d$ is greater than the wire radius $r_{a}\left(d \gg r_{a}\right) \cdot \rho_{i}=\left(Z_{i}-Z_{c}\right) /\left(Z_{i}+Z_{c}\right)(i=1,2)$ are the reflection coefficients at each node of the line. $L$ is the length of the line. $Z_{1}$ and $Z_{2}$ are the load impedances at the ends $x=x_{0}$ and $x=x_{0}+L$, respectively, and $Z_{c}=120 \ln \left(d / r_{a}\right)$ is the characteristic impedance of the line. The transmission line theory shows that the wave propagation constant $\gamma=j k_{0}$. 
In this paper, we only consider the transmission line mode current. The BLT equation of the load currents and the total voltages at the loads can be expressed in the matrix form as

$$
\begin{gathered}
{\left[\begin{array}{c}
I\left(x_{0}\right) \\
I\left(x_{0}+L\right)
\end{array}\right]=\frac{1}{Z_{c}}\left[\begin{array}{cc}
1-\rho_{1} & 0 \\
0 & 1-\rho_{2}
\end{array}\right]\left[\begin{array}{cc}
-\rho_{1} & e^{\gamma L} \\
e^{\gamma L} & -\rho_{2}
\end{array}\right]^{-1}\left[\begin{array}{l}
S_{1} \\
S_{2}
\end{array}\right],} \\
{\left[\begin{array}{c}
V\left(x_{0}\right) \\
V\left(x_{0}+L\right)
\end{array}\right]=\left[\begin{array}{cc}
1+\rho_{1} & 0 \\
0 & 1+\rho_{2}
\end{array}\right]\left[\begin{array}{cc}
-\rho_{1} & e^{\gamma L} \\
e^{\gamma L} & -\rho_{2}
\end{array}\right]^{-1}\left[\begin{array}{l}
S_{1} \\
S_{2}
\end{array}\right] .}
\end{gathered}
$$

We choose the Taylor formulation (see $[14,15]$ ), since it consists of both voltage and current sources that are appropriate for localized excitations through apertures and the source vector given by

$$
\left[\begin{array}{l}
S_{1} \\
S_{2}
\end{array}\right]=\left[\begin{array}{c}
\frac{1}{2} \int_{x_{0}}^{x_{0}+L} e^{\gamma x}\left[V_{S}(x)+Z_{c} I_{S}(x)\right] d x \\
-\frac{1}{2} \int_{x_{0}}^{x_{0}+L} e^{\gamma(L-x)}\left[V_{S}(x)-Z_{c} I_{S}(x)\right] d x
\end{array}\right],
$$

in which voltage and current sources $V_{S}(x)$ and $I_{S}(x)$ are given by

$$
\begin{gathered}
V_{S}(x)=-j \omega \mu_{0} \int_{z_{0}}^{z_{0}+d} H_{y}^{b}\left(x, y_{0}, z\right) d z, \\
I_{S}(x)=-j \omega C \int_{z_{0}}^{z_{0}+d} E_{z}^{b}\left(x, y_{0}, z\right) d z,
\end{gathered}
$$

where

$$
C=\frac{\pi \varepsilon}{\ln \left(d / r_{a}\right)} \quad\left(d \gg r_{a}\right)
$$

Substituting (3.38) into (4.4) and (3.39) into (4.3) then into (4.2) can determine the source vector. Thereafter, using the BLT equation, we obtain the induced voltage and current of a two-wire line at each load in a multiaperture cavity under excitation.

\section{Simulation}

We present some numerical examples to demonstrate the formulation given in the previous section. We consider the coupling in a cavity with two rectangular apertures on the plane $z=0$. There is a two-wire transmission line along the $x$ direction in this cavity, see Figure 1. We excite the cavity by a plane wave incident normal to the side of the box on which two apertures resides. The parameters are as follows: the cavity's size $a=0.3 \mathrm{~m}, b=0.3 \mathrm{~m}, c=$ $0.2 \mathrm{~m}$, two identical apertures' size $l=0.1 \mathrm{~m}, w=0.005 \mathrm{~m}$, the location of the first aperture $(0.15 \mathrm{~m}, 0.12 \mathrm{~m}, 0 \mathrm{~m})$, and the location of the second aperture $(0.15 \mathrm{~m}, 0.18 \mathrm{~m}, 0 \mathrm{~m})$, the wire 


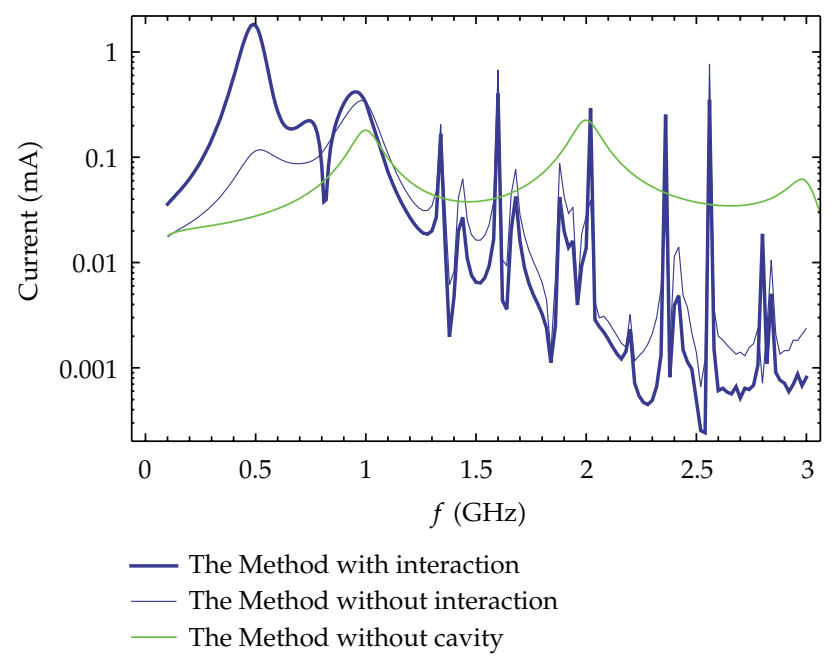

Figure 5: Comparison of induced currents of a two-wire line at load under excitation.

radius $r_{a}=0.0003 \mathrm{~m}$, the wire length $L=0.15 \mathrm{~m}$, the wire separation distance $d=0.02 \mathrm{~m}$, the characteristic impedance $Z_{c} \approx 503 \Omega$, the load resistance $Z_{1}=Z_{2}=50 \Omega$.

The two-wire transmission line can be excited by the interior coupling electromagnetic field. By employing the BLT equation, Figure 5 shows the results of the terminal response at Junction 4 with those parameters. This example indicates that the effect of the external coupling fields on the two-wire line current depends on both the multiaperture cavity and the electromagnetic interaction among apertures. More precisely, the difference between the results with the electromagnetic interaction and those without the electromagnetic interaction is actually not small at all. Therefore, this electromagnetic interaction cannot be simply neglected for the frequency range up to $1 \mathrm{GHz}$. Moreover, using the BLT equation, we can also conclude that the currents and voltages at arbitrary point on the two-wire line can be derived from the interior coupling EM fields.

\section{Conclusion}

In this paper, the field coupling phenomenon between external fields and a two-wire line in the multiaperture cavity is studied. For the issue of the field penetration through apertures, the EMT theory and the multistep iteration are used to deal with these problems. This method can also be used for electromagnetic interaction problems on more complex system. Then, using the Modal Green's Function and the method of moments, the electromagnetic total coupling fields are determined inside a multiaperture cavity. Finally, the load response of the two-wire transmission line could be resolved by the BLT equation. The results with the electromagnetic interaction are largely different from those without the electromagnetic interaction for the low frequency.

\section{Acknowledgments}

This work was supported by China Postdoctoral Special Science Foundation (no. 200902662), the National Natural Science Foundation of China (no. 10871231), and Pre-research Foundation of Weapon and Equipment (no. 9140A31020609KG0170). 


\section{References}

[1] T. Konefal, J. F. Dawson, and A. C. Marvin, "Improved aperture model for shielding prediction," in Proceedings of the IEEE Symposium on Electromagnetic Compatibility, pp. 187-192, August 2003.

[2] G. Cerri, R. de Leo, and V. M. Primiani, "Theoretical and experimental evaluation of the electromagnetic radiation from apertures in shielded enclosures," IEEE Transactions on Electromagnetic Compatibility, vol. 34, no. 4, pp. 423-432, 1992.

[3] M. D. Deshpande, "Electromagnetic field penetration studies," Tech. Rep., NASA, June 2000.

[4] F. C. Yang and C. E. Baum, "Use of matrix norms of interaction supermatrix blocks for specifying electromagnetic performance of subshields," Interaction Notes 427, 1983.

[5] P. Kirawanich, R. Gunda, N. S. Kranthi, J. C. Kroenung, and N. E. Islam, "Methodology for interference analysis using electromagnetic topology techniques," Applied Physics Letters, vol. 84, no. 15, pp. 2949-2951, 2004.

[6] J. E. Nanevicz, E. F. Vance, W. Radasky, M. A. Uman, G. K. Soper, and J. Ma Pierre, “EMP susceptibility insights from aircraft exposure to lightning," IEEE Transactions on Electromagnetic Compatibility, vol. 30, no. 4, pp. 463-472, 1988.

[7] J. D. Turner, T. M. Benson, C. Christopoulos et al., "Characterization of the shielding effectiveness of equipment cabinets containing apertures," in Proceedings of IEEE Electromagnetic Compatibility Conference, pp. 574-578, 1996.

[8] J. P. Parmantier, "Numerical coupling models for complex systems and results," IEEE Transactions on Electromagnetic Compatibility, vol. 46, no. 3, pp. 359-367, 2004.

[9] F. M. Tesche and C. M. Bulter, "On the addition of EM field propagation and coupling effects in the BLT equation," Interaction Notes 588, June 2004.

[10] P. Kirawanich, J. Wilson, N. E. Islam, C. Christodoulou, and S. J. Yakura, "Electromagnetic topology: a modular junction approach for a system level interaction problem," in Proceedings of the IEEE International Symposium on Electromagnetic Compatibility (EMC'07), July 2007.

[11] T. Yang, Coupling onto radio frequency components enclosed within canonical structures, A dissertation for the Ph.D., University of Michigan, Ann Arbor, Mich, USA, 2006.

[12] K. Barkeshli and J. L. Volakis, "Electromagnetic scattering from an aperture formed by a rectangular cavity recessed in a ground plane," Journal of Electromagnetic Waves and Application, vol. 5, pp. 715-734, 1991.

[13] P. Kirawanich, N. S. Kranthi, and N. E. Islam, "Modeling external interference on systems using electromagnetic topology technique," in Proceedings of the International Symposium on Electromagnetic Compatibility (EMC '04), vol. 3, pp. 804-808, Santa Clara, Calif, USA, August 2004.

[14] C. D. Taylor, R. S. Scatterwhite, and C. W. Harrison, "The response of a terminated two-Wires transmission-line excited by a nonuniform electromagnetic field," IEEE Transactions on Antennas and Propagation, vol. 13, pp. 987-989, 1965.

[15] F. M. Tesche, M. V. Ianoz, and T. Karlsson, EMC: Analysis Methods and Computational Models, John Wiley \& Sons, New York, NY, USA, 1997. 


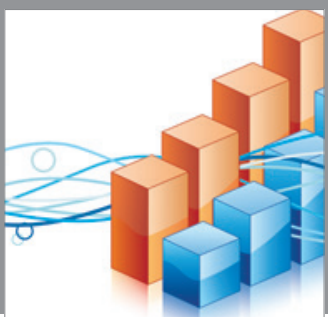

Advances in

Operations Research

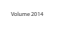

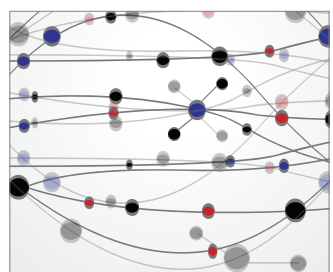

\section{The Scientific} World Journal
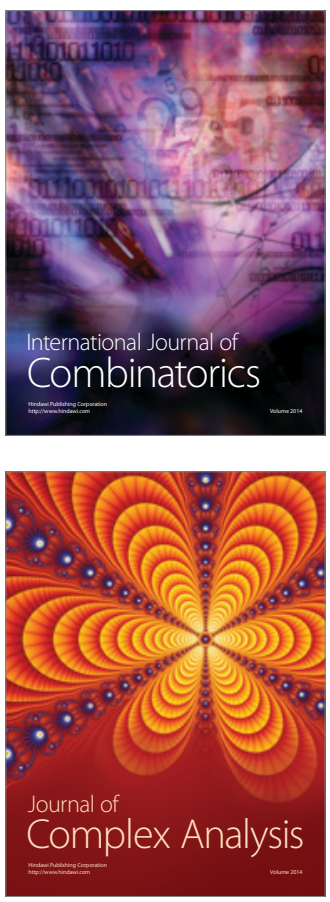

International Journal of

Mathematics and

Mathematical

Sciences
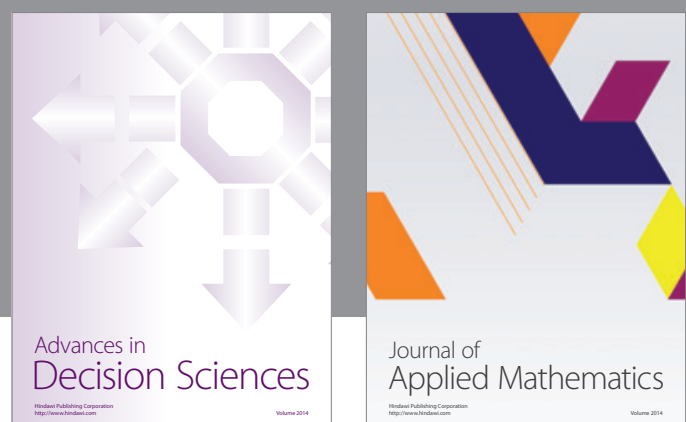

Journal of

Applied Mathematics
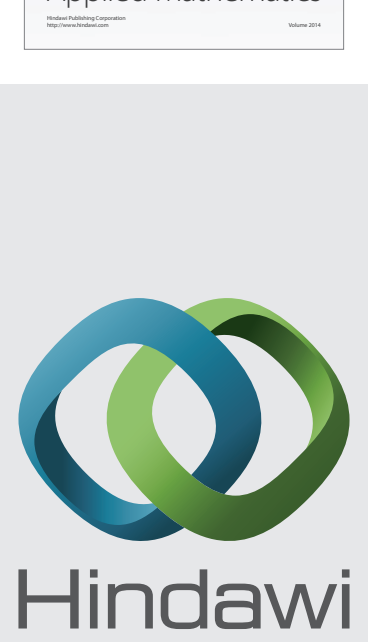

Submit your manuscripts at http://www.hindawi.com
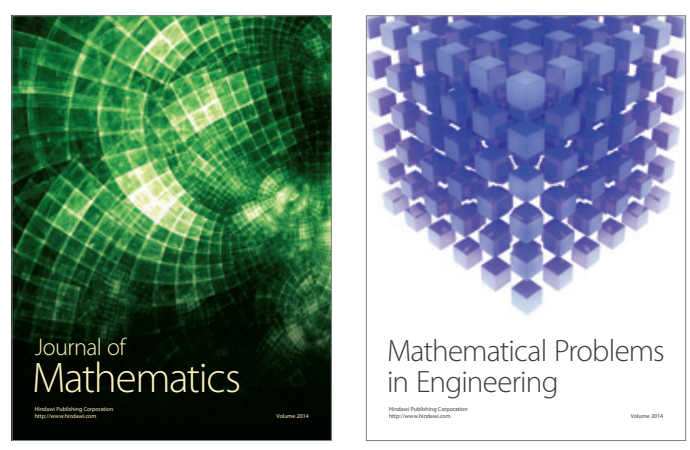

Mathematical Problems in Engineering
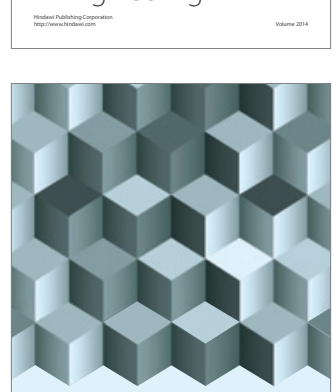

Journal of

Function Spaces
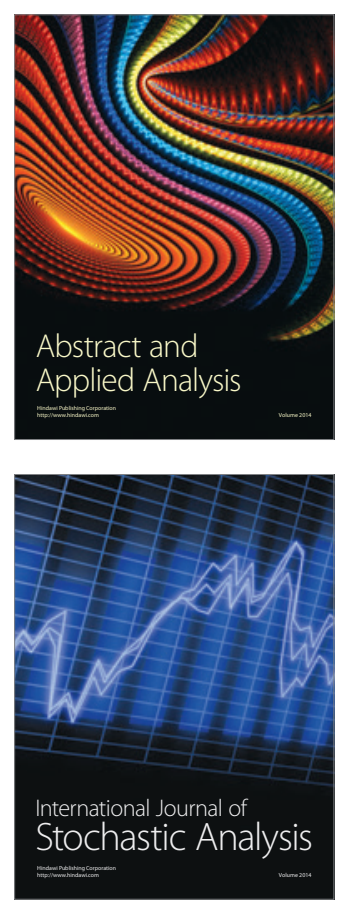

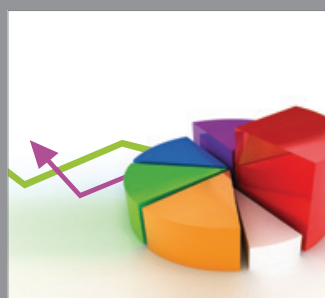

ournal of

Probability and Statistics

Promensencen
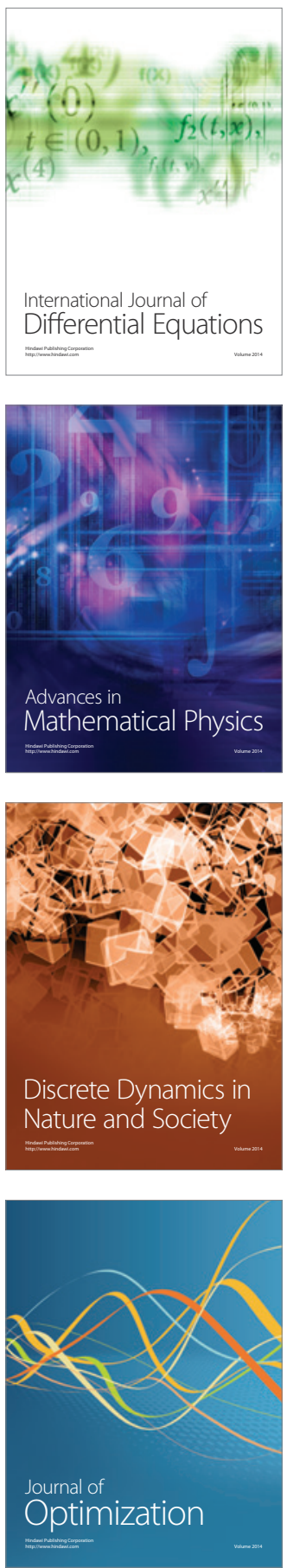